\title{
A Step by Step Guide on Derivation and Analysis of a New Numerical Method for Solving Fourth-order Ordinary Differential Equations
}

\author{
Ezekiel Olaoluwa Omole ${ }^{1}$, Luke Azeta Ukpebor ${ }^{2}$ \\ ${ }^{1}$ Department of Mathematics \& Statistics, College of Natural Sciences, Joseph Ayo Babalola University, Osogbo, Nigeria \\ ${ }^{2}$ Department of Mathematics, Faculty of Physical Sciences, Ambrose Alli University, Ekpoma, Nigeria
}

Email address:

omolez247@gmail.com (E. O. Omole), eoomole@jabu.edu.ng (E. O. Omole), lukeukpebor@gmail.com (L. A. Ukpebor)

\section{To cite this article:}

Ezekiel Olaoluwa Omole, Luke Azeta Ukpebor. A Step by Step Guide on Derivation and Analysis of a New Numerical Method for Solving Fourth-order Ordinary Differential Equations. Mathematics Letters. Vol. 6, No. 2, 2020, pp. 13-31. doi: 10.11648/j.ml.20200602.12

Received: April 25, 2020; Accepted: May 18, 2020; Published: September 23, 2020

\begin{abstract}
This manuscript presents a step by step guide on derivation and analysis of a new numerical method to solve initial value problem of fourth order ordinary differential equations. The method adopted hybrid techniques using power series as the basic function. Collocation of the fourth derivatives was done at both grid and off-grid points. The interpolation of the approximate function is also taken at the first four points. The complete derivation of the new technique is introduced and shown here, as well as the full analysis of the method. The discrete schemes and its first, second, and third derivatives were combined together and solved simultaneously to obtain the required 32 family of block integrators. The block integrators are then applied to solve problem. The method was tested on a linear system of equations of fourth order ordinary differential equation in order to check the practicability and reliability of the proposed method. The results are displaced in tables; it converges faster and uses smaller time for its computations. The basic properties of the method were examined, the method has order of accuracy $p=10$, the method is zero stable, consistence, convergence and absolutely stable. In future study, we will investigate the feasibility, convergence, and accuracy of the method by on some standard complex boundary value problems of fourth order ordinary differential equations. The extension of this new numerical method will be illustrated and comparison will also be made with some existing methods.
\end{abstract}

Keywords: Derivation, Analysis, Fourth-order Ordinary Differential Equations, New numerical Method, Hybrid Techniques, Convergence, Zero Stability, Consistency, Taylor Series, Order 10, Integrators

\section{Introduction}

This work proposes the derivation and analysis of a new numerical method for direct approximation of the differential equations of the form.

$$
y^{\prime \prime \prime \prime}=f\left(x, y, y^{\prime}, y^{\prime \prime}, y^{\prime \prime \prime}\right), y\left(x_{0}\right)=\tau_{0}, y^{\prime}\left(x_{0}\right)=\tau_{1}, y^{\prime \prime}\left(x_{0}\right)=\tau_{2}, y^{\prime \prime \prime}\left(x_{0}\right)=\tau_{3}(1)
$$

where $R \times R^{m} \times R^{m} \rightarrow R^{m}$ and $y, y_{0}, y^{\prime}, y^{\prime \prime}, y^{\prime \prime \prime} \in \mathfrak{R}$ are given real constants.

Several authors have devoted a lot of attention to the development of various methods for solving (1) directly without reducing it to system of first order. Numerous numerical methods based on the use of different polynomial functions and non polynomials have been adopted including
Power series [1], Legendre and Chebyshev [2], Taylor series [3], power series and exponential functions [4], Legendre polynomial [5], Adomian decomposition [6], Bernstein Polynomial Basis [8], Lucas Polynomial [9] and, Finite-Difference [12, 14].

In this paper, we are motivated by the work of [1] to develop an hybrid block method with four-step and four-hybrid points with characteristic of high order for solving directly fourth order ordinary differential equations. According to [1], power series has great properties, easy to use and gives a better performance. This work is motivated by the need to develop a new numerical method which can handle system of equations of initial value problems of fourth order ordinary differential equations. The new method is expected to converge faster with lesser time of computation. Hence address setback 
associated with other methods in the literature. The method has order of accuracy $\mathrm{P}=10$, the accessibly and accuracy of the methods shall be illustrated on the table.

\section{Description of the Method}

The section deals with the step by step derivation of the new numerical method for solution of initial value problems of fourth order ordinary differential equations.

\subsection{Derivation of the Method}

The approximate solution of the form

$$
y(x)=\sum_{j=0}^{12} a_{j} x^{j}
$$

is considered for numerical approximation of (1). Where $a_{j}$ are parameters to be determined and $k=4$ See [1-4] for more details. The first, second, third and fourth derivatives of (2) are

$$
y^{\prime}(x)=\sum_{j=0}^{12} j a_{j} x^{j-1}
$$

$$
y^{\prime \prime}(x)=\sum_{j=0}^{12} j(j-1) a_{j} x^{j-2}
$$

$$
\begin{gathered}
y^{\prime \prime \prime}(x)=\sum_{j=0}^{12} j(j-2)(j-1) a_{j} x^{j-3} \\
y^{i v}(x)=\sum_{j=0}^{12} j(j-3)(j-2)(j-1) a_{j} x^{j-4}
\end{gathered}
$$

Substituting (6) into (2) to obtain

$$
\sum_{j=0}^{12} j(j-3)(j-2)(j-1) a_{j} x^{j-4}=f\left(x, y, y^{\prime}, y^{\prime \prime}, y^{\prime \prime \prime}\right)(7)
$$

\begin{tabular}{|c|c|c|c|c|c|c|c|c|c|c|c|c|c|c|}
\hline 1 & $x_{n}$ & $x_{n}^{2}$ & $x_{n}^{3}$ & $x_{n}^{4}$ & $x_{n}^{5}$ & $x_{n}^{6}$ & $x_{n}^{7}$ & $x_{n}^{8}$ & $x_{n}^{9}$ & $x_{n}^{10}$ & $x_{n}^{11}$ & $x_{n}^{12}$ & & \\
\hline & $x_{n+\frac{1}{2}}$ & $x^{2} n+\frac{1}{2}$ & $x^{3} n+\frac{1}{2}$ & $x_{n+\frac{1}{2}}^{4}$ & $x^{5}+\frac{1}{2}$ & $x_{n+\frac{1}{2}}^{6}$ & $\mathrm{x}_{\mathrm{n}+\frac{1}{2}}^{7}$ & $x_{n+\frac{1}{2}}^{8}$ & $x^{9}+\frac{1}{2}$ & $x^{10}+\frac{1}{2}$ & $x^{11}+\frac{1}{2}$ & $x_{n+\frac{1}{2}}^{12}$ & & $y$ \\
\hline & $x_{n+1}$ & $x_{n+1}^{2}$ & $x_{n+1}^{3}$ & $x_{n+1}^{4}$ & $x_{n+1}^{5}$ & $x_{n+1}^{6}$ & $x_{n+1}^{7}$ & $x_{n+1}^{8}$ & $x_{n+1}^{9}$ & $x_{n+1}^{10}$ & $x_{n+1}^{11}$ & $x_{n+1}^{12}$ & $a_{0}$ & \\
\hline & $x_{n+\frac{3}{2}}$ & $x^{2}$ & $x_{n+\frac{3}{2}}^{3}$ & $x^{4}+\frac{3}{2}$ & $x^{5}+\frac{3}{2}$ & $x^{6}+\frac{3}{2}$ & $x^{7} n+\frac{3}{2}$ & $x_{n+\frac{3}{2}}^{8}$ & $x_{n+\frac{3}{2}}^{9}$ & $x_{n+\frac{3}{2}}^{10}$ & $x^{11} n+\frac{3}{2}$ & $x^{12} n+\frac{3}{2}$ & $\begin{array}{l}a_{1} \\
a_{2}\end{array}$ & $\begin{array}{l}y_{n+1} \\
y_{n+\frac{3}{2}}\end{array}$ \\
\hline 0 & 0 & 0 & 0 & 24 & $120 x_{n}$ & $360 x_{n}^{2}$ & $840 x_{n}^{3}$ & $1680 x_{n}^{4}$ & $3024 x_{n}^{5}$ & $5040 x_{n}^{6}$ & $7920 x_{n}^{7}$ & $11880 x_{n}^{8}$ & $a_{3}$ & \\
\hline 0 & 0 & 0 & 0 & 24 & $120 x$ & $360 x^{2} n+\frac{1}{2}$ & $840 x^{3} n+\frac{1}{2}$ & $1680 x^{4} n+\frac{1}{2}$ & $\frac{3024 x^{5}}{n+\frac{1}{2}}$ & $5040 x^{6} n+\frac{1}{2}$ & ${ }^{7920 x^{7}}+\frac{1}{2}$ & $11880 x^{8} n+\frac{1}{2}$ & $\begin{array}{l}a_{4} \\
a_{5}\end{array}$ & $f$ \\
\hline 0 & 0 & 0 & 0 & 24 & $120 x_{n+1}$ & $360 x_{n+1}^{2}$ & $840 x_{n+1}^{3}$ & $1680 x_{n+1}^{4}$ & $3024 x_{n+1}^{5}$ & $5040 x_{n+1}^{6}$ & $7920 x_{n+1}^{7}$ & $11880 x_{n+1}^{8}$ & $a_{6}=$ & $=f_{n+1}$ \\
\hline 0 & 0 & 0 & 0 & 24 & $120 x n+\frac{3}{2}$ & $\begin{array}{r}360 x^{2} \\
n+\frac{3}{2}\end{array}$ & $840 x^{3} n+\frac{3}{2}$ & $1680 x^{4} n+\frac{3}{2}$ & $3024 x^{5} n+\frac{3}{2}$ & $\begin{array}{r}5040 x^{6} \\
n+\frac{3}{2}\end{array}$ & $\begin{array}{r}7920 x^{7} \\
n+\frac{3}{2}\end{array}$ & $11880 x^{8} n+\frac{3}{2}$ & $a_{7}$ & \\
\hline 0 & 0 & 0 & 0 & 24 & $120 x_{n+2}$ & $360 x_{n+2}^{2}$ & $840 x_{n+2}^{3}$ & $1680 x_{n+2}^{4}$ & $3024 x_{n+2}^{5}$ & $5040 x_{n}^{6}$ & $+27920 x_{t}$ & $x_{n+2}^{7} \quad 11880 x_{n+2}^{8}$ & $a_{9}$ & \\
\hline 0 & 0 & 0 & 0 & 24 & $120 x_{n+\frac{5}{2}}$ & $360 x^{2} n+\frac{5}{2}$ & $840 x^{3} n+\frac{5}{2}$ & $1680 x^{4} n+\frac{5}{2}$ & $3024 x^{5} n+\frac{5}{2}$ & $5040 x^{6} n+\frac{5}{2}$ & $7920 x^{7} n+\frac{5}{2}$ & $11880 x^{8} n+\frac{5}{2}$ & $\begin{array}{l}a_{10} \\
a_{11}\end{array}$ & \\
\hline 0 & 0 & 0 & 0 & 24 & $120 x_{n+2}$ & $360 x_{n+2}^{2}$ & $840 x_{n+2}^{3}$ & $1680 x_{n+2}^{4}$ & $3024 x_{n+2}^{5}$ & $5040 x_{n}^{6}$ & $+27920 x$ & $c_{n+2}^{7} \quad 11880 x_{n+2}^{8}$ & {$\left[\begin{array}{l}11 \\
a_{12}\end{array}\right]$} & \\
\hline 0 & 0 & 0 & 0 & 24 & $120 x n+\frac{7}{2}$ & $360 x^{2} n+\frac{7}{2}$ & $\begin{array}{r}840 x^{3} \\
n+\frac{7}{2}\end{array}$ & $1680 x^{4}$ & $3024 x^{5} n+\frac{7}{2}$ & $5040 x^{6} n+\frac{7}{2}$ & $\frac{7920 x^{7}}{n+\frac{7}{2}}$ & $11880 x^{8} n+\frac{7}{2}$ & & $f$ \\
\hline & 0 & 0 & 0 & 24 & $120 x_{n+4}$ & $360 x_{n+4}^{2}$ & $840 x_{n+4}^{3}$ & $1680 x_{n+4}^{4}$ & $3024 x_{n+4}^{5}$ & $5040 x_{n}^{6}$ & $7920 x$ & $11880 x_{n+4}^{8}$ & & \\
\hline
\end{tabular}

where $C=$ Points of collocation, $I=$ Points of interpolation and $E=$ Point of evaluation. $C+I-1=12$ Collocate equation (6) at $x=x_{n+i}, i=0, \frac{1}{2}, 1, \frac{3}{2}, 2, \frac{5}{2}, 3, \frac{7}{2}, 4$, Interpolate (2) at $x=x_{n+i}, i=0, \frac{1}{2}, 1, \frac{3}{2}$ and evaluating at the end point $x=x_{n+i}, i=4$. This can be represented in matrix as follows,
Solve for $a_{j}, j=0(1) 12$ in (8) using Gaussian elimination method with the help of Computer Aided Software gives:

$$
y(x)=\sum_{j=0}^{k-1} \alpha_{j}(x) y_{n+j}+h^{4}\left(\sum_{j=0}^{k} \beta_{j}(x) f_{n+j}+\beta_{v}(x) f_{n+v}\right)(9)
$$

where $y(x)$ is the numerical solution of the initial value problem and $v=\frac{1}{2}, \frac{3}{2}, \frac{5}{2}, \frac{7}{2} \cdot \alpha_{\mathrm{j}}$ and $\beta_{\mathrm{j}}$ are constants.

$$
f_{n+j}=f\left(x_{n+j}, y_{n+j}, y_{n+j}^{\prime}, y_{n+j}^{\prime \prime}, y_{n+j}^{\prime \prime}\right) \text {, }
$$

using the transformation $t=\frac{x-x_{n+3}}{h}, \frac{d t}{d x}=\frac{1}{h}$

The coefficients of $y_{n+j}$ and $f_{n+j}$ are obtained as:

$$
\alpha_{0}(t)=\frac{-1}{3}\left(-3+36 t+24 t^{2}+4 t^{3}\right), \quad \alpha_{\frac{1}{2}}(t)=\left(36+54 t+26 t^{2}+4 t^{3}\right),
$$




$$
\begin{aligned}
& \alpha_{1}(t)=\left(-45-63 t-28 t^{2}-4 t^{3}\right), \alpha_{\frac{3}{2}}(t)=\frac{1}{3}\left(60+74 t+30 t^{2}+4 t^{3}\right) \\
& \beta_{0}(t)=\frac{h^{4}}{3832012800}\left[\begin{array}{l}
-836550-1171485 t-565061 t^{2}-249876 t^{3}+380160 t^{5}+198528-228096 t^{6} \\
-228096 t^{7}-209088 t^{8}+50688 t^{10}+18432 t^{11}+2048 t^{12}
\end{array}\right] \\
& \beta_{\frac{1}{2}}(t)=\frac{-h^{4}}{479001600}\left[\begin{array}{l}
-49289130-77044707 t-39299207 t^{2}-6737984 t^{3}+456192 t^{5}+228096 t^{6} \\
-278784 t^{7}-240768 t^{8}+57040 t^{10}+19968 t^{11}+2048 t^{12}
\end{array}\right] \\
& \beta_{1}(t)=\frac{h^{4}}{958003200}\left[\begin{array}{l}
447663150+726110865 t+386383357 t^{2}+65955296 t^{3}+3991680 t^{5}+1862784 t^{6} \\
-2496384 t^{7}-1970496 t^{8}+140800 t^{9}+489984 t^{10}+150528 t^{11}+14336 t^{12}
\end{array}\right] \\
& \beta_{2}(t)=\frac{h^{4}}{383201280}\left[\begin{array}{l}
40333590+108979461 t+101714005 t^{2}+29214988 t^{3}+7983360 t^{5}+2395008 t^{6} \\
-5220864 t^{7}-2578752 t^{8}+619520 t^{9}+692736 t^{10}+172032 t^{11}+14336 t^{12}
\end{array}\right] \\
& \beta_{3}(t)=\frac{h^{4}}{958003200}\left[\begin{array}{l}
9386001+5584590 t+14988421 t^{2}+33454872 t^{3}+39916800 t^{4}+15168384 t^{5}-9787008 t^{6} \\
-10378368 t^{7}-1463616 t^{8}+1774080 t^{9}+963072 t^{10}+193536 t^{11}+14336 t^{12}
\end{array}\right] \\
& \beta_{\frac{7}{2}}(t)=\frac{h^{4}}{479001600}\left[\begin{array}{l}
-678150-1012725 t-787081 t^{2}-921448 t^{3}+2280960 t^{5}+2963248 t^{6} \\
+1343232 t^{7}-139392 t^{8}-387200 t^{9}-160512 t^{10}-29184 t^{11}-2048 t^{12}
\end{array}\right] \\
& \beta_{4}(t)=\frac{h^{4}}{383201280}\left[\begin{array}{l}
589050+873315 t+577003 t^{2}+537020 t^{3}-1140480 t^{5}-1140480 t^{6}+ \\
126720 t^{7}+804672 t^{8}+563200 t^{9}+185856 t^{10}+30720 t^{11}+2048 t^{12}
\end{array}\right]
\end{aligned}
$$

Evaluating (10) at $\mathrm{t}=1$ gives the discrete scheme below

$$
y_{n+4}+140 y_{n+1}-120 y_{n+\frac{1}{2}}-56 y_{n+\frac{3}{2}}+35 y_{n}=\frac{h^{4}}{829440}\left[\begin{array}{c}
-565 f_{n}+298040 f_{n+\frac{1}{2}}+1409780 f_{n+1}+ \\
1022792 f_{n+\frac{3}{2}}+615410 f_{n+2}+192200 f_{n+\frac{5}{2}} \\
+86420 f_{n+3}+4280 f_{n+\frac{7}{2}}+443 f_{n+4}
\end{array}\right]
$$

First derivative of (10) gives

$$
\begin{gathered}
\alpha_{0}^{\prime}(t)=\frac{-1}{3 h}\left(36+48 t+12 t^{2}\right), \alpha_{\frac{1}{2}}^{\prime}(t)=\frac{1}{h}\left(54+52 t+12 t^{2}\right), \alpha_{1}^{\prime}(t)=\frac{1}{h}\left(-63-84 t-12 t^{2}\right), \alpha_{\frac{3}{2}}^{\prime}(t)=\frac{1}{3 h}\left(74+60 t+12 t^{2}\right) \\
\beta_{0}^{\prime}(t)=\frac{h^{3}}{3832012800}\left[\begin{array}{l}
-1171485-1130122 t-749628 t^{2}+1900800 t^{4}+1191168 t^{5}- \\
156672 t^{6}-1672704 t^{7}+506880 t^{9}+202752 t^{10}+24576 t^{11}
\end{array}\right] \\
\beta_{\frac{1}{2}}^{\prime}(t)=\frac{h^{3}}{479001600}\left[\begin{array}{l}
-77044707-78598414 t-20213952 t^{2}+2280960 t^{4}+1368576 t^{5}- \\
1951488 t^{6}-1926144 t^{7}+63360 t^{8}+591360 t^{9}+219648 t^{10}+124576 t^{11}
\end{array}\right] \\
\beta_{1}^{\prime}(t)=\frac{h^{3}}{958003200}\left[\begin{array}{l}
726110865+77276670 t+19786588 t^{2}+19958400 t^{4}+11176704 t^{5}-17474688 t^{6} \\
-15763968 t^{7}+1267200 t^{8}+4899840 t^{9}+1655808 t^{10}+172032 t^{11}
\end{array}\right]
\end{gathered}
$$




$$
\begin{gathered}
\beta_{\frac{3}{2}}^{\prime}(t)=\frac{-h^{3}}{479001600}\left[\begin{array}{l}
-254454165-345068816 t-123540120 t^{2}+26611200 t^{4}+13128192 t^{5}- \\
23950080 t^{6}-18551808 t^{7}+2851200 t^{8}+5829120 t^{9}+1774080 t^{10}+172032 t^{11}
\end{array}\right] \\
\beta_{2}^{\prime}(t)=\frac{h^{3}}{3832012800}\left[\begin{array}{l}
108979461+203428010 t+87644964 t^{2}+39916800 t^{4}+14370048 t^{5}- \\
36546048 t^{6}-20630016 t^{7}+5575680 t^{9}+1892352 t^{10}+172032 t^{11}
\end{array}\right] \\
\beta_{\frac{5}{2}}^{\prime}(t)=\frac{-h^{3}}{479001600}\left[\begin{array}{l}
-18799965-109771954 t-139107936 t^{2}+79833600 t^{4}-3193344 t^{5}- \\
55528704 t^{6}-19565568 t^{7}+9820800 t^{8}+8194560 t^{9}-2010624 t^{10}+172032 t^{11}
\end{array}\right] \\
\beta_{3}^{\prime}(t)=\frac{h^{3}}{958003200}\left[\begin{array}{l}
9386001+29976842 t+100364616 t^{2}+159667200 t^{3}+75841920 t^{4}-58722048 t^{5} \\
-72648576 t^{6}-11708928 t^{7}+15966720 t^{8}+9630720 t^{9}+2128896 t^{10}+172032 t^{11}
\end{array}\right] \\
\beta_{\frac{7}{2}}^{\prime}(t)=\frac{h^{3}}{479001600}\left[\begin{array}{l}
-1012725-1574162 t-2764344 t^{2}+11404800 t^{4}+17779488 t^{5}+ \\
9402624 t^{6}-1115136 t^{7}-3484800 t^{8}-1605120 t^{9}-321024 t^{10}-24576 t^{11}
\end{array}\right] \\
\beta_{4}^{\prime}(t)=\frac{h^{3}}{3832012800}\left[\begin{array}{l}
873315+1154006 t+1611060 t^{2}-5702400 t^{4}-6614784 t^{5}+ \\
887040 t^{6}+6437376 t^{7}+5068800 t^{8}+1858560 t^{9}+337920 t^{10}+24576 t^{11}
\end{array}\right]
\end{gathered}
$$

while the second derivative of (10) gives:

$$
\begin{gathered}
\alpha_{0}^{\prime \prime}(t)=\frac{-1}{3 h^{2}}(48+24 t), \alpha_{\frac{1}{2}}^{\prime \prime}(t)=\frac{1}{h^{2}}(52+24 t), \alpha_{1}^{\prime \prime}(t)=\frac{1}{h^{2}}(-84-24 t), \alpha_{\frac{3}{2}}^{\prime \prime}(t)=\frac{1}{3 h^{2}}(60+24 t) \\
\beta_{0}^{\prime \prime}(t)=\frac{h^{2}}{3832012800}\left[\begin{array}{l}
-1130122-1499256 t+7603200 t^{3}+5955840 t^{4}-940032 t^{5}- \\
11708928 t^{6}+4561920 t^{8}+2027520 t^{9}+270336 t^{10}
\end{array}\right] \\
\beta_{\frac{1}{2}}^{\prime \prime}(t)=\frac{-h^{2}}{479001600}\left[\begin{array}{l}
-78598414-40427904 t+9123840 t^{3}+6842880 t^{4}-11708928 t^{5} \\
-13483008 t^{6}+506880 t^{7}+5322240 t^{8}+2196480 t^{9}+270336 t^{10}
\end{array}\right] \\
\beta_{1}^{\prime \prime}(t)=\frac{h^{2}}{958003200}\left[\begin{array}{l}
77276670+395731776 t+79833600 t^{3}+55883520 t^{4}-104848128 t^{5} \\
-110247776 t^{6}+10137600 t^{7}+44098560 t^{8}+16558080 t^{9}+1892352 t^{10}
\end{array}\right] \\
\beta_{\frac{3}{2}}^{\prime \prime}(t)=\frac{-h^{2}}{479001600}\left[\begin{array}{l}
-345068816-247080240 t+106444800 t^{3}+65640960 t^{4}-143700480 t^{5} \\
-129862656 t^{6}+22809600 t^{7}+52462080 t^{8}+17740800 t^{9}+1892352 t^{10}
\end{array}\right] \\
\beta_{\frac{3}{2}}^{\prime \prime}(t)=\frac{-h^{2}}{479001600}\left[\begin{array}{l}
-345068816-247080240 t+106444800 t^{3}+65640960 t^{4}-143700480 t^{5} \\
-129862656 t^{6}+22809600 t^{7}+52462080 t^{8}+17740800 t^{9}+1892352 t^{10}
\end{array}\right] \\
\beta_{2}^{\prime \prime}(t)=\frac{h^{2}}{3832012800}\left[\begin{array}{l}
203428010+175289928 t+159667200 t^{3}+71850240 t^{4}-219276288 t^{5}- \\
144410112 t^{6}+44605440 t^{7}+62346240 t^{8}+18923520 t^{9}+1892352 t^{10}
\end{array}\right] \\
\beta_{\frac{5}{2}}^{\prime \prime}(t)=\frac{-h^{2}}{479001600}\left[\begin{array}{l}
-109771954-278215872 t+319334400 t^{3}-15966720 t^{4}-333172224 t^{5} \\
-136958976 t^{6}+78566400 t^{7}+73751040 t^{8}-20106240 t^{9}+1892352 t^{10}
\end{array}\right] \\
\beta_{3}^{\prime \prime}(t)=\frac{h^{2}}{958003200}\left[\begin{array}{l}
29976842+200729232 t+479001600 t^{2}+303367680 t^{3}-293610240 t^{4} \\
-435891456 t^{5}-81962496 t^{6}+127733760 t^{8}+2188960 t^{9}+1892352 t^{10}
\end{array}\right] \\
\beta_{\frac{7}{2}}^{\prime \prime}(t)=\frac{-h^{2}}{479001600}\left[\begin{array}{l}
-1574162-5528688 t+45619200 t^{3}+88897440 t^{4}+56415744 t^{5} \\
-7805952 t^{6}-27878400 t^{7}-14446080 t^{8}-3210240 t^{9}-270336 t^{10}
\end{array}\right] \\
\beta_{4}^{\prime \prime}(t)=\frac{h^{2}}{3832012800}\left[\begin{array}{l}
1154006+3222120 t-22809600 t^{3}-33073920 t^{4}+5322240 t^{5} \\
+45061632 t^{6}+40550400 t^{8}+16727040 t^{8}+3379200 t^{9}+270336 t^{10}
\end{array}\right]
\end{gathered}
$$

while the third derivative of (10) gives: 


$$
\begin{aligned}
& \alpha_{0}^{\prime \prime \prime}(t)=\frac{-8}{h^{3}}, \alpha_{1 / 2}^{\prime \prime \prime}(t)=\frac{24}{h^{3}}, \alpha_{1}^{\prime \prime \prime}(t)=\frac{-24}{h^{3}}, \alpha_{3 / 2}^{\prime \prime \prime}(t)=\frac{24}{h^{3}} \\
& \beta_{0}^{\prime \prime \prime}(t)=\frac{h}{3832012800}\left[\begin{array}{l}
-1499256+22809600 t^{2}+23823360 t^{3}-4700160 t^{4} \\
-70253568 t^{5}+36495360 t^{7}+18247680 t^{8}+2703360 t^{9}
\end{array}\right] \\
& \beta_{\frac{1}{2}}^{\prime \prime \prime}(t)=\frac{-h}{479001600}\left[\begin{array}{l}
-404277904+27371520 t^{2}+27371520 t^{3}-58544640 t^{4}-80898048 t^{5} \\
+3548160 t^{6}+42577920 t^{7}+19768320 t^{8}+2703360 t^{9}
\end{array}\right] \\
& \beta_{1}^{\prime \prime \prime}(t)=\frac{h}{958003200}\left[\begin{array}{l}
395731776+239500800 t^{2}+223534080 t^{3}-524240640 t^{4}-662086656 t^{5} \\
+70963200 t^{6}+352788480 t^{7}+149022720 t^{8}+18923520 t^{9}
\end{array}\right] \\
& \beta_{\frac{3}{2}}^{\prime \prime \prime}(t)=\frac{-h}{479001600}\left[\begin{array}{l}
-247080240+319334400 t^{2}+262563840 t^{3}-718502400 t^{4}-779175936 t^{5} \\
+159667200 t^{6}+419696640 t^{7}+159667200 t^{8}+18923520 t^{9}
\end{array}\right] \\
& \beta_{2}^{\prime \prime \prime}(t)=\frac{h}{3832012800}\left[\begin{array}{l}
175289928+479001600 t^{2}+287400960 t^{3}-1096381440 t^{4} \\
-866460672 t^{5}+312238080 t^{6}+498769920 t^{7}+170311680 t^{8}
\end{array}\right] \\
& \beta_{\frac{5}{2}}^{\prime \prime \prime}(t)=\frac{-h}{479001600}\left[\begin{array}{l}
-278215872+958003200 t^{2}-63866880 t^{3}-1665861120 t^{4}-821753856 t^{5} \\
+549964800 t^{6}+408320 t^{7}-180956160 t^{8}+18923520 t^{9}
\end{array}\right] \\
& \beta_{3}^{\prime \prime \prime \prime}(t)=\frac{h}{958003200}\left[\begin{array}{l}
200729232+958003200 t+910103040 t^{2}-1174440960 t^{3}-2179457280 t^{4} \\
-4917749 t^{5}+894136320 t^{6}+693411840 t^{7}+19700640 t^{8}+18923520 t^{9}
\end{array}\right] \\
& \beta_{7}^{\prime \prime \prime \prime}(t)=\frac{h}{2^{3}}\left[\begin{array}{l}
-5528688+136857600 t^{2}+355589760 t^{3}+282078720 t^{4}-46835712 t^{5} \\
-195148800 t^{6}-115568640 t^{7}-28892160 t^{8}-2703360 t^{9}
\end{array}\right] \\
& \beta_{4}^{\prime \prime \prime}(t)=\frac{h}{3832012800}\left[\begin{array}{l}
3222120-68428800 t^{2}-132295680 t^{3}+26611200 t^{4}+270369792 t^{5} \\
+324403200 t^{7}+133816320 t^{7}+30412800 t^{8}+2703360 t^{9}
\end{array}\right]
\end{aligned}
$$

Evaluating (10) at non-interpolate points at $t=0,-1, \frac{-1}{2} 2$ gives the following discrete schemes

$$
\begin{aligned}
& y_{n+3}-20 y_{n+\frac{3}{2}}+45 y_{n+1}-36 y_{n+\frac{1}{2}}+10 y_{n}=\frac{-h^{4}}{3870720}\left[\begin{array}{c}
845 f_{n}-398296 f_{n+\frac{1}{2}}^{-1808740 f_{n+1}-} \\
1012840 f_{n+\frac{3}{2}}^{-407410 f_{n+2}+15320 f_{n+\frac{5}{2}}} \\
-22564 f_{n+3}+5480 f_{n+\frac{7}{2}}^{-595 f_{n+4}}
\end{array}\right] \\
& y_{n+2}-4 y_{n+\frac{3}{2}}+6 y_{n+1}-4 y_{n+\frac{1}{2}}+y_{n}=\frac{-h^{4}}{11612160}\left[\begin{array}{c}
329 f_{n}-120280 f_{n+\frac{1}{2}}-492004 f_{n+1}- \\
100648 f_{n+\frac{3}{2}}-24922 f_{n+2}+17624 f_{n+\frac{5}{2}} \\
-7492 f_{n+3}+1832 f_{n+\frac{7}{2}}-199 f_{n+4}
\end{array}\right]
\end{aligned}
$$




$$
\begin{aligned}
& y_{n+\frac{5}{2}}-10 y_{n+\frac{3}{2}}+20 y_{n+1}-15 y_{n+\frac{1}{2}}+4 y_{n}=\frac{-h^{4}}{11612160}\left[\begin{array}{c}
117 f_{n}-47900 f_{n+\frac{1}{2}}^{-2095460 f_{n+1}-} \\
877880 f_{n+\frac{3}{2}}^{-225410 f_{n+2}+70648 f} \\
-29060 f_{n+3}+700 f_{n+\frac{7}{2}}{ }^{-755 f_{n+4}}
\end{array}\right] \\
& y_{n+\frac{7}{2}}-35 y_{n+\frac{3}{2}}+84 y_{n+1}-70 y_{n+\frac{1}{2}}+20 y_{n}=\frac{-h^{4}}{1658880}\left[\begin{array}{c}
685 f_{n}-341000 f_{n+\frac{1}{2}}-1585604 f_{n+1}- \\
1041320 f_{n+\frac{3}{2}}^{-537410 f_{n+2}-92120 f_{n+\frac{5}{2}}} \\
-3626 f_{n+3}+4744 f_{n+\frac{7}{2}}-515 f_{n+4}
\end{array}\right]
\end{aligned}
$$

Evaluating (12) at points $t=-3, \frac{-5}{2},-2, \frac{-3}{2}, 0,-1, \frac{-1}{2} 2$ gives

$$
\begin{aligned}
& 1277337600 h y_{n}^{\prime}+4683571200 y_{n}+851558400 y_{n+\frac{3}{2}}-3832012800 y_{n+1}+7664025600 y_{n+\frac{1}{2}} \\
& =h^{4}\left[\begin{array}{l}
-1322633 f_{n}+30018920 f_{n+\frac{1}{2}}-6069500 f_{n+1}+851558400 f_{n+\frac{3}{2}}+6551290 f_{n+2}-4396952 f_{n+\frac{5}{2}} \\
+1873060 f_{n+3}-464360 f_{n+\frac{7}{2}}+51175 f_{n+4}
\end{array}\right] \\
& 3832012800 y_{n+\frac{1}{2}}^{\prime}+2554675200 y_{n}+1277337600 y_{n+\frac{3}{2}}-7664025600 y_{n+1}+3832012800 y_{n+\frac{1}{2}} \\
& =h^{4}\left[\begin{array}{l}
81145 f_{n}+21111976 f_{n+\frac{1}{2}}+23711020 f_{n+1}+1277337600 f_{n+\frac{3}{2}}+6467110 f_{n+2}-3567560 f_{n+\frac{5}{2}} \\
+1353484 f_{n+3}-310760 f_{n+\frac{7}{2}}+32425 f_{n+4}
\end{array}\right] \\
& 3832012800 h y_{n+1}^{\prime}-1277337600 y_{n}-2554675200 y_{n+\frac{3}{2}}-3832012800 y_{n+1}+7664025600 y_{n+\frac{1}{2}} \\
& =h^{4}\left[\begin{array}{l}
68615 f_{n}-13441480 f_{n+\frac{1}{2}}-31841164 f_{n+1}+2554675200 f_{n+\frac{3}{2}}-761910 f_{n+2}+4320200 f_{n+\frac{5}{2}} \\
-1667980 f_{n+3}+387704 f_{n+\frac{7}{2}}-40825 f_{n+4}
\end{array}\right] \\
& 1277337600 y_{n+\frac{3}{2}}^{\prime}+851558400 y_{n}+4683571200 y_{n+\frac{3}{2}}+7664025600 y_{n+1}+3832012800 y_{n+\frac{1}{2}} \\
& =h^{4}\left[\begin{array}{l}
37735 f_{n}+8965880 f_{n+\frac{1}{2}}+31109900 f_{n+1}+4683571200 f_{n+\frac{3}{2}}+3418310 f_{n+2}-2117080 f_{n+\frac{5}{2}} \\
+86420 f_{n+3}-200440 f_{n+\frac{7}{2}}+21353 f_{n+4}
\end{array}\right]
\end{aligned}
$$




$$
\begin{aligned}
& 1277337600 y_{n+2}^{\prime}+14050713600 y_{n}-33210777600 y_{n+\frac{3}{2}}+72808243200 y_{n+1}-53648179200 y_{n+\frac{1}{2}} \\
& =h^{4}\left[\begin{array}{l}
-334031 f_{n}+144849064 f_{n+\frac{1}{2}}+624528604 f_{n+1}+33210777600 f_{n+\frac{3}{2}}+31957750 f_{n+2}- \\
19141544 f_{n+\frac{5}{2}}+8095036 f_{n+3}-19983704 f_{n+\frac{7}{2}}+216001 f_{n+4}
\end{array}\right]
\end{aligned}
$$

$$
\begin{aligned}
& 3832012800 y_{n+\frac{7}{2}}^{\prime}+94522982400 y_{n}-136675123200 y_{n+\frac{3}{2}}+360209203200 y_{n+1}-318057062400 y_{n+\frac{1}{2}} \\
& =h^{4}\left[\begin{array}{l}
-1804745 f_{n}+970047880 f_{n+\frac{1}{2}}+4652709556 f_{n+1}+36550359720 f_{n+\frac{3}{2}}+2348536090 f_{n+2}+ \\
836297560 f_{n+\frac{5}{2}}+284743060 f_{n+3}-8807816 f_{n+\frac{7}{2}}+1377895 f_{n+4}
\end{array}\right] \\
& 3832012800 h y_{n+4}^{\prime}+136675123200 y_{n}+186491289600 y_{n+\frac{3}{2}}-501993676800 y_{n+1}+452177510400 y_{n+\frac{1}{2}} \\
& =h^{4}\left[\begin{array}{c}
-2494435 f_{n}+1401489800 f_{n+\frac{1}{2}}+6810539180 f_{n+1}+5721593336 f_{n+\frac{3}{2}}+4117306430 f_{n+2}+ \\
1967486840 f_{n+\frac{5}{2}}+1040221580 f_{n+3}+213576200 f_{n+\frac{7}{2}}+5935469 f_{n+4}
\end{array}\right]
\end{aligned}
$$

Evaluating (13) at $t=-3, \frac{-5}{2},-2, \frac{-3}{2}, 0,-1, \frac{-1}{2} 2$ gives

$$
\begin{aligned}
& 1916006400 h^{2} y_{n}^{\prime \prime}-15328051200 y_{n}+7664025600 y_{n+\frac{3}{2}}-30656102400 y_{n+1}+3832012800 y_{n+\frac{1}{2}} \\
& =h^{4}\left[\begin{array}{l}
29397487 f_{n}+371214904 f_{n+\frac{1}{2}}-40557452 f_{n+1}+172563976 f_{n+\frac{3}{2}}-164939750 f_{n+2} \\
+105890248 f_{n+\frac{5}{2}}-44086604 f_{n+3}+10778872 f_{n+\frac{7}{2}}-1176881 f_{n+4}
\end{array}\right]
\end{aligned}
$$

$1916006400 h^{2} y_{n+\frac{1}{2}}^{\prime \prime}-7664025600 y_{n}+8127128 y_{n+\frac{3}{2}}-7664025600 y_{n+1}+15328051200 y_{n+\frac{1}{2}}$ $=h^{4}\left[\begin{array}{l}-959741 f_{n}-39395384 f_{n+\frac{1}{2}}+4124548 f_{n+1}-8127128 f_{n+\frac{3}{2}}+7828450 f_{n+2}-5020904 f_{n+\frac{5}{2}} \\ +2087524 f_{n+3}-509768 f_{n+\frac{7}{2}}+55603 f_{n+4}\end{array}\right]$ 


$$
\begin{aligned}
& 1916006400 h^{2} y_{n+1}^{\prime \prime}+0 y_{n}-7664025600 y_{n+\frac{3}{2}}+15328051200 y_{n+1}+1460168 y_{n+\frac{1}{2}} \\
& =\frac{h^{4}}{123344}\left[\begin{array}{l}
55603 f_{n}-1460168 f_{n+\frac{1}{2}}-37393676 f_{n+1}-546104 f_{n+\frac{3}{2}}-1121150 f_{n+2} \\
+822472 f_{n+\frac{5}{2}}-350252 f_{n+3}+85816 f_{n+\frac{7}{2}}-9341 f_{n+4}
\end{array}\right]
\end{aligned}
$$

$1916006400 h^{2} y_{n+\frac{3}{2}}^{\prime \prime}+7664025600 y_{n}-15328051200 y_{n+\frac{3}{2}}+3832012800 y_{n+1}-30656102400 y_{n+\frac{1}{2}}$ $=h^{4}\left[\begin{array}{l}-226481 f_{n}+79524472 f_{n+\frac{1}{2}}+322926196 f_{n+1}+29818648 f_{n+\frac{3}{2}}+14725450 f_{n+2}- \\ 11576024 f_{n+\frac{5}{2}}+4982548 f_{n+3}-1223096 f_{n+\frac{7}{2}}+133087 f_{n+4}\end{array}\right]$

$1916006400 h^{2} y_{n+2}^{\prime \prime}+15328051200 y_{n}-22992076800 y_{n+\frac{3}{2}}+61312204800 y_{n+1}-53648179200 y_{n+\frac{1}{2}}$ $=h^{4}\left[\begin{array}{l}-301193 f_{n}+157345336 f_{n+\frac{1}{2}}+733760884 f_{n+1}+444602248 f_{n+\frac{3}{2}}+79484650 f_{n+2}- \\ 25307192 f_{n+\frac{5}{2}}+9492724 f_{n+3}-2226824 f_{n+\frac{7}{2}}+237367 f_{n+4}\end{array}\right]$

$1916006400 h^{2} y_{n+\frac{5}{2}}^{\prime \prime}+22992076800 y_{n}+30656102400 y_{n+\frac{3}{2}}+84304281600 y_{n+1}-76640256000 y_{n+\frac{1}{2}}$ $=h^{4}\left[\begin{array}{l}-414053 f_{n}+235716904 f_{n+\frac{1}{2}}+1140058468 f_{n+1}+913105096 f_{n+\frac{3}{2}}+523856050 f_{n+2}+ \\ 14680888 f_{n+\frac{5}{2}}+9465796 f_{n+3}-2679848 f_{n+\frac{7}{2}}+303499 f_{n+4}\end{array}\right]$

$1916006400 h^{2} y_{n+3}^{\prime \prime}+30656102400 y_{n}-3832012800 y_{n+\frac{3}{2}}+107296358400 y_{n+1}-99632332800 y_{n+\frac{1}{2}}$ $=h^{4}\left[\begin{array}{l}-565061 f_{n}+314393656 f_{n+\frac{1}{2}}+1545533428 f_{n+1}+1380275272 f_{n+\frac{3}{2}}+1017140050 f_{n+2}+ \\ 439087816 f_{n+\frac{5}{2}}+59953684 f_{n+3}-6296648 f_{n+\frac{7}{2}}+577003 f_{n+4}\end{array}\right]$

$1916006400 h^{2} y_{n+\frac{7}{2}}^{\prime \prime}+3832012800 y_{n}-45984153600 y_{n+\frac{3}{2}}+130288435200 y_{n+1}-122624409600 y_{n+\frac{1}{2}}$ $=h^{4}\left[\begin{array}{l}-508697 f_{n}+391165912 f_{n+\frac{1}{2}}+1958778964 f_{n+1}+1829203576 f_{n+\frac{3}{2}}+1535220250 f_{n+2}+ \\ 886278472 f_{n+\frac{5}{2}}+512279668 f_{n+3}+33135976 f_{n+\frac{7}{2}}-446921 f_{n+4}\end{array}\right]$

$1916006400 h^{2} y_{n+4}^{\prime \prime}+45984153600 y_{n}-53648179200 y_{n+\frac{3}{2}}+153280512000 y_{n+1}-145616486400 y_{n+\frac{1}{2}}$ $=h^{4}\left[\begin{array}{l}-1749761 f_{n}+479822392 f_{n+\frac{1}{2}}+2323412596 f_{n+1}+2394886408 f_{n+\frac{3}{2}}+1871582650 f_{n+2}+ \\ 1521741256 f_{n+\frac{5}{2}}+878405428 f_{n+3}+521114104 f_{n+\frac{7}{2}}+29901727 f_{n+4}\end{array}\right]$

Evaluating (14) at $t=-3, \frac{-5}{2},-2, \frac{-3}{2}, 0,-1, \frac{-1}{2} 2$ gives 


$$
\begin{aligned}
& 14515200 h^{3} y_{n}^{\prime \prime \prime}+116121600 y_{n}-116121600 y_{n+\frac{3}{2}}+348364800 y_{n+1}-348364800 y_{n+\frac{1}{2}} \\
& =h^{4}\left[\begin{array}{l}
-2084463 f_{n}+38364800 f_{n+\frac{1}{2}}+5902624 f_{n+1}-9225936 f_{n+\frac{3}{2}}+8506010 f_{n+2}- \\
5379392 f_{n+\frac{5}{2}}+22222 f_{n+3}-540784 f_{n+\frac{7}{2}}+58861 f_{n+4}
\end{array}\right]
\end{aligned}
$$

$14515200 h^{3} y_{n+\frac{1}{2}}^{\prime \prime \prime}+116121600 y_{n}-116121600 y_{n+\frac{3}{2}}+348364800 y_{n+1}-348364800 y_{n+\frac{1}{2}}$ $=h^{4}\left[\begin{array}{l}55571 f_{n}-1411412 f_{n+\frac{1}{2}}-3306564 f_{n+1}+116121600 f_{n+\frac{3}{2}}+1560230 f_{n+2}+913284 f_{n+\frac{5}{2}} \\ -360148 f_{n+3}+84964 f_{n+\frac{7}{2}}-9045 f_{n+4}\end{array}\right]$

$14515200 h^{3} y_{n+1}^{\prime \prime \prime}+116121600 y_{n}-116121600 y_{n+\frac{3}{2}}+348364800 y_{n+1}-348364800 y_{n+\frac{1}{2}}$

$=h^{4}\left[\begin{array}{l}-12335 f_{n}+1339776 f_{n+\frac{1}{2}}+3183008 f_{n+1}+116121600 f_{n+\frac{3}{2}}+1074330 f_{n+2}-596800 f_{n+\frac{5}{2}} \\ +228424 f_{n+3}-52848 f_{n+\frac{7}{2}}+5549 f_{n+4}\end{array}\right]$

$14515200 h^{3} y_{n+\frac{3}{2}}^{\prime \prime \prime}+116121600 y_{n}-116121600 y_{n+\frac{3}{2}}+348364800 y_{n+1}-348364800 y_{n+\frac{1}{2}}$

$=h^{4}\left[\begin{array}{l}2259 f_{n}+348364800 f_{n+\frac{1}{2}}+6459580 f_{n+1}+3723372 f_{n+\frac{3}{2}}-591910 f_{n+2}+198916 f_{n+\frac{5}{2}} \\ -55764 f_{n+3}+10340 f_{n+\frac{7}{2}}-917 f_{n+4}\end{array}\right]$

$14515200 h^{3} y_{n+2}^{\prime \prime \prime}+116121600 y_{n}-116121600 y_{n+\frac{3}{2}}+348364800 y_{n+1}-348364800 y_{n+\frac{1}{2}}$

$=h^{4}\left[\begin{array}{l}-4207 f_{n}+1213312 f_{n+\frac{1}{2}}+6027552 f_{n+1}+7543088 f_{n+\frac{3}{2}}+3857050 f_{n+2}-652608 f_{n+\frac{5}{2}} \\ +196808 f_{n+3} 41072 f_{n+\frac{7}{2}}+4077 f_{n+4}\end{array}\right]$

$14515200 h^{3} y_{n+\frac{5}{2}}^{\prime \prime \prime}+116121600 y_{n}-116121600 y_{n+\frac{3}{2}}+348364800 y_{n+1}-348364800 y_{n+\frac{1}{2}}$

$=h^{4}\left[\begin{array}{l}787 f_{n}+1161900 f_{n+\frac{1}{2}}+6280124 f_{n+1}+6691564 f_{n+\frac{3}{2}}+8306010 f_{n+2}+3167108 f_{n+\frac{5}{2}} \\ 235220 f_{n+3}+31716 f_{n+\frac{7}{2}}-2389 f_{n+4}\end{array}\right]$

$14515200 h^{3} y_{n+3}^{\prime \prime \prime}+116121600 y_{n}-116121600 y_{n+\frac{3}{2}}+348364800 y_{n+1}-348364800 y_{n+\frac{1}{2}}$

$=h^{4}\left[\begin{array}{l}-5679 f_{n}+1225088 f_{n+\frac{1}{2}}+5995936 f_{n+1}+7487280 f_{n+\frac{3}{2}}+6639770 f_{n+2}+8430784 f_{n+\frac{5}{2}} \\ +3041352 f_{n+3}-167536 f_{n+\frac{7}{2}}+12205 f_{n+4}\end{array}\right]$

$14515200 h^{3} y_{n+\frac{7}{2}}^{\prime \prime \prime}+116121600 y_{n}-116121600 y_{n+\frac{3}{2}}+348364800 y_{n+1}-348364800 y_{n+\frac{1}{2}}$

$=h^{4}\left[\begin{array}{l}8915 f_{n}+1087276 f_{n+\frac{1}{2}}+6584508 f_{n+1}+5977196 f_{n+\frac{3}{2}}+9274330 f_{n+2}+4925700 f_{n+\frac{5}{2}} \\ +9530924 f_{n+3}+2583652 f_{n+\frac{7}{2}}-55701 f_{n+4}\end{array}\right]$ 


$$
\begin{aligned}
& 14515200 h^{3} y_{n+4}^{\prime \prime \prime}+116121600 y_{n}-116121600 y_{n+\frac{3}{2}}+348364800 y_{n+1}-348364800 y_{n+\frac{1}{2}} \\
& =h^{4}\left[\begin{array}{l}
-58991 f_{n}+1713024 f_{n+\frac{1}{2}}+4002080 f_{n+1}+12269872 f_{n+\frac{3}{2}}-791910 f_{n+2}+16116416 f_{n+\frac{5}{2}} \\
+321736 f_{n+3}+11517840 f_{n+\frac{7}{2}}+2084333 f_{n+4}
\end{array}\right]
\end{aligned}
$$

\subsection{Derivation of the Block Integrators}

This is done by combining equations (11), (15), (16), (17), (18), (19), (28) and (37) and solve simultaneously to give block integrators which will be used in the future research to solve complex problems.

$$
\left[\begin{array}{llllllll}
1 & 0 & 0 & 0 & 0 & 0 & 0 & 0 \\
0 & 1 & 0 & 0 & 0 & 0 & 0 & 0 \\
1 & 0 & 1 & 0 & 0 & 0 & 0 & 0 \\
0 & 0 & 0 & 1 & 0 & 0 & 0 & 0 \\
0 & 0 & 0 & 0 & 1 & 0 & 0 & 0 \\
0 & 0 & 0 & 0 & 0 & 1 & 0 & 0 \\
0 & 0 & 0 & 0 & 0 & 0 & 1 & 0 \\
0 & 0 & 0 & 0 & 0 & 0 & 0 & 1
\end{array}\right]\left[\begin{array}{l}
y_{n+\frac{1}{2}} \\
y_{n+1} \\
y_{n+\frac{3}{2}} \\
y_{n+2} \\
y_{n+\frac{5}{2}} \\
y_{n+3} \\
y_{n+\frac{7}{2}} \\
y_{n+4}
\end{array}\right]=\left[\begin{array}{llllllll}
0 & 0 & 0 & 0 & 0 & 0 & 0 & 1 \\
0 & 0 & 0 & 0 & 0 & 0 & 0 & 1 \\
0 & 0 & 0 & 0 & 0 & 0 & 0 & 1 \\
0 & 0 & 0 & 0 & 0 & 0 & 0 & 1 \\
0 & 0 & 0 & 0 & 0 & 0 & 0 & 1 \\
0 & 0 & 0 & 0 & 0 & 0 & 0 & 1 \\
0 & 0 & 0 & 0 & 0 & 0 & 0 & 1 \\
0 & 0 & 0 & 0 & 0 & 0 & 0 & 1
\end{array}\right]\left[\begin{array}{l}
y_{n-\frac{1}{2}} \\
y_{n-1} \\
y_{n-\frac{3}{2}} \\
y_{n-2} \\
y_{n-\frac{5}{2}} \\
y_{n-3} \\
y_{n-\frac{7}{2}} \\
y_{n}
\end{array}\right]+
$$

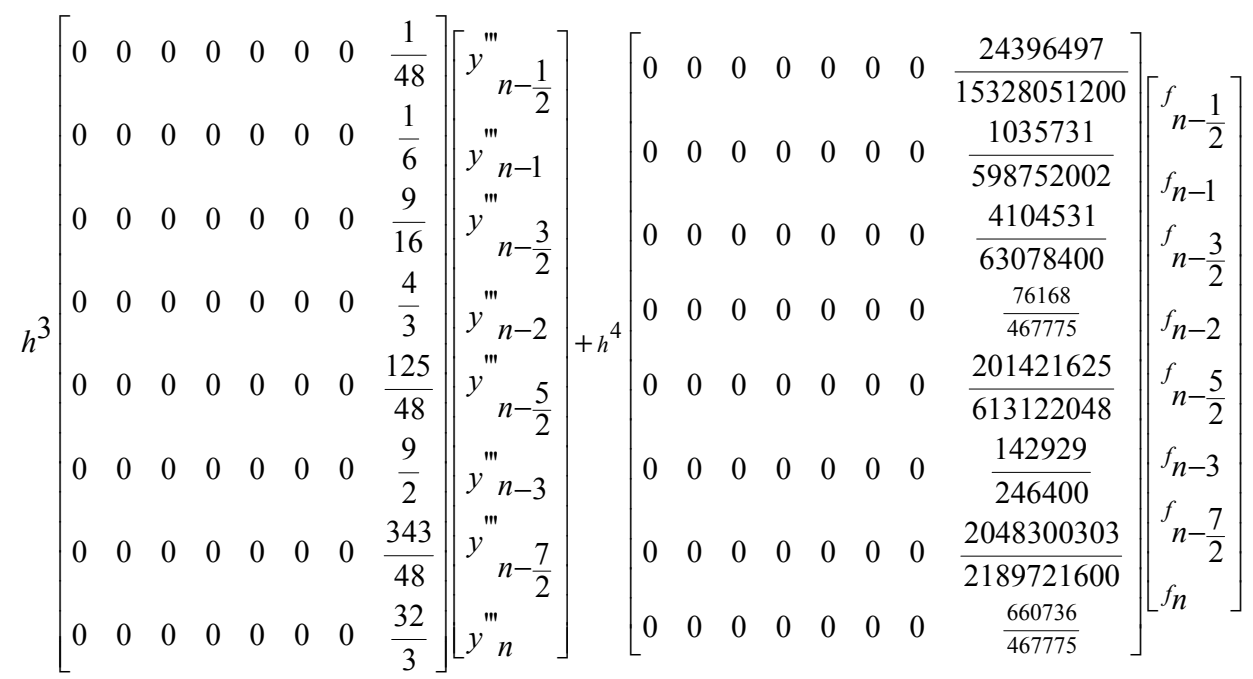

$+h^{4}\left[\begin{array}{ccccccccc}\frac{1520909}{638668800} & \frac{-13220819}{3832012800} & \frac{8390797}{19600640} & \frac{-2050007}{510935040} & \frac{4854761}{1916006400} & \frac{-364589}{348364800} & \frac{162689}{63866880} & \frac{-42511}{15328051200} \\ \frac{169969}{3742200} & \frac{-37379}{712800} & \frac{245837}{3742200} & \frac{357779}{5987520} & \frac{46873}{1247400} & \frac{-231691}{1496880} & \frac{14071}{3742200} & \frac{-8159}{19958400} \\ \frac{156411}{716800} & \frac{-3119229}{15769600} & \frac{59337}{225280} & \frac{-1516887}{6307840} & \frac{1194183}{7884800} & \frac{-984537}{15769600} & \frac{17091}{1126400} & \frac{-20817}{12615680} \\ \frac{96704}{155925} & \frac{-3119229}{15769600} & \frac{320704}{466775} & \frac{-50}{81} & \frac{181952}{467775} & \frac{-75032}{467775} & \frac{1216}{31185} & \frac{-1984}{467775} \\ \frac{103514375}{76640256} & \frac{-41912}{93555} & \frac{111998125}{76640256} & \frac{-383340625}{366561024} & \frac{2901125}{3649536} & \frac{-50269375}{153280512} & \frac{79375}{995328} & \frac{-1773125}{204374016} \\ \frac{38737}{15400} & \frac{-77193}{61600} & \frac{42057}{15400} & \frac{-53217}{24640} & \frac{21951}{15400} & \frac{-5139}{8800} & \frac{2187}{15400} & \frac{-3807}{246400} \\ \frac{38267583}{91238400} & \frac{-89766187}{49766400} & \frac{1268609167}{273715200} & \frac{-244827569}{72990720} & \frac{650716619}{273715200} & \frac{-512596693}{547430400} & \frac{21001547}{91238400} & \frac{54841241}{2189721600} \\ \frac{3043228}{467775} & \frac{-381952}{155925} & \frac{684032}{93555} & \frac{-302946223}{47900160} & \frac{5348}{14175} & \frac{-631808}{467775} & \frac{167936}{467775} & \frac{-1184}{31185}\end{array}\right]\left[\begin{array}{l}f_{n+\frac{1}{2}} \\ f_{n+1} \\ f_{n+\frac{3}{2}} \\ f_{n+2} \\ f_{n+\frac{5}{2}} \\ f_{n+3} \\ f_{n+\frac{7}{2}}^{7} \\ f_{n+4}\end{array}\right]$

Writing out (46) explicitly as follow 


$$
\begin{aligned}
& y_{n+\frac{1}{2}}=y_{n}-\frac{1}{2} h y_{n}^{\prime}+\frac{1}{8} h^{2} y_{n}^{\prime \prime}+\frac{1}{48} h^{3} y_{n}^{\prime \prime \prime}+h^{4}\left(\begin{array}{l}
\frac{24396497}{15328051200} f_{n}+\frac{1520909}{638668800} f_{n+\frac{1}{2}}-\frac{13220819}{3832012800} f_{n+1} \\
+\frac{8390797}{191600640} f_{n+\frac{3}{2}}-\frac{2050007}{510935040} f_{n+2}+\frac{4854761}{1916006400} f_{n+\frac{5}{2}} \\
-\frac{364589}{348364800} f_{n+3}+\frac{162689}{638668800} f_{n+\frac{7}{2}}-\frac{425111}{15328051200} f_{n+4}
\end{array}\right) \\
& y_{n+1}=y_{n}-h y_{n}^{\prime}+\frac{1}{2} h^{2} y_{n}^{\prime \prime}+\frac{1}{6} h^{3} y_{n}^{\prime \prime \prime}+h^{4}\left(\begin{array}{l}
\frac{1035731}{59875200} f_{n}+\frac{169969}{3742200} f_{n+\frac{1}{2}}-\frac{37379}{712800} f_{n+1} \\
+\frac{245837}{3742200} f_{n+\frac{3}{2}}-\frac{357779}{5987520} f_{n+2}+\frac{46873}{1247400} f_{n+\frac{5}{2}} \\
-\frac{231691}{1496880} f_{n+3}+\frac{14071}{3742200} f_{n+\frac{7}{2}}-\frac{8159}{19958400} f_{n+4}
\end{array}\right) \\
& y_{n+\frac{3}{2}}=y_{n}-\frac{3}{2} h y_{n}^{\prime}+\frac{9}{8} h^{2} y_{n}^{\prime \prime}+\frac{9}{16} h^{3} y_{n}^{\prime \prime \prime}+h^{4}\left(\begin{array}{l}
\frac{4104531}{63078400} f_{n}+\frac{156411}{716800} f_{n+\frac{1}{2}}-\frac{3119229}{15769600} f_{n+1} \\
+\frac{59337}{225280} f_{n+\frac{3}{2}}-\frac{1516887}{6307840} f_{n+2}+\frac{1194183}{7884800} f_{n+\frac{5}{2}} \\
-\frac{984537}{15769600} f_{n+3}+\frac{17091}{1126400} f_{n+\frac{7}{2}}-\frac{20817}{12615680} f_{n+4}
\end{array}\right) \\
& y_{n+2}=y_{n}-2 h y_{n}^{\prime}+2 h^{2} y_{n}^{\prime \prime}+\frac{4}{3} h^{3} y_{n}^{\prime \prime \prime}+h^{4}\left(\begin{array}{l}
\frac{76168}{467775} f_{n}+\frac{96704}{155925} f_{n+\frac{1}{2}}-\frac{41912}{93555} f_{n+1} \\
+\frac{320704}{467775} f_{n+\frac{3}{2}}-\frac{50}{81} f_{n+2}+\frac{181952}{467775} f_{n+\frac{5}{2}} \\
-\frac{75032}{467775} f_{n+3}+\frac{1216}{31185} f_{n+\frac{7}{2}}-\frac{1984}{467775} f_{n+4}
\end{array}\right) \\
& y_{n+\frac{5}{2}}=y_{n}-\frac{5}{2} h y_{n}^{\prime}+\frac{25}{8} h^{2} y_{n}^{\prime \prime}+\frac{125}{48} h^{3} y_{n}^{\prime \prime \prime}+h^{4}\left(\begin{array}{l}
\frac{201421625}{613122048} f_{n}+\frac{103514375}{76640256} f_{n+\frac{1}{2}}-\frac{40900625}{51093504} f_{n+1} \\
+\frac{111998125}{76640256} f_{n+\frac{3}{2}}-\frac{383340625}{306561024} f_{n+2}+\frac{2901125}{3649536} f_{n+\frac{5}{2}} \\
-\frac{50269375}{153280512} f_{n+3}+\frac{79375}{995328} f_{n+\frac{7}{2}}-\frac{1773125}{204374016} f_{n+4}
\end{array}\right) \\
& y_{n+3}=y_{n}-3 h y_{n}^{\prime}+\frac{9}{2} h^{2} y_{n}^{\prime \prime}+\frac{9}{2} h^{3} y_{n}^{\prime \prime \prime}+h^{4}\left(\begin{array}{l}
\frac{142929}{246400} f_{n}+\frac{38637}{15400} f_{n+\frac{1}{2}}-\frac{77193}{61600} f_{n+1} \\
+\frac{42057}{15400} f_{n+\frac{3}{2}}-\frac{53217}{24640} f_{n+2}+\frac{21951}{15400} f_{n+\frac{5}{2}} \\
-\frac{5139}{8800} f_{n+3}+\frac{2187}{15400} f_{n+\frac{7}{2}}-\frac{3807}{246400} f_{n+4}
\end{array}\right)
\end{aligned}
$$




$$
\begin{aligned}
& y_{n+\frac{7}{2}}=y_{n}-\frac{7}{2} h y_{n}^{\prime}+\frac{49}{8} h^{2} y_{n}^{\prime \prime}+\frac{343}{48} h^{3} y_{n}^{\prime \prime \prime}+h^{4}\left(\begin{array}{l}
\frac{2048300303}{2189721600} f_{n}+\frac{38267583}{91238400} f_{n+\frac{1}{2}}-\frac{89766187}{49766400} f_{n+1} \\
+\frac{1268609167}{273715200} f_{n+\frac{3}{2}}-\frac{244827569}{72990720} f_{n+2}+\frac{650716619}{273715200} f_{n+\frac{5}{2}} \\
-\frac{512596693}{547430400} f_{n+3}+\frac{21001547}{91238400} f_{n+\frac{7}{2}}-\frac{54841241}{2189721600} f_{n+4}
\end{array}\right) \\
& y_{n+4}=y_{n}-4 h y_{n}^{\prime}+8 h^{2} y_{n}^{\prime \prime}+\frac{32}{3} h^{3} y_{n}^{\prime \prime \prime}+h^{4}\left(\begin{array}{l}
\frac{660736}{467775} f_{n}+\frac{3043228}{467775} f_{n+\frac{1}{2}}-\frac{381952}{155925} f_{n+1} \\
+\frac{684032}{93555} f_{n+\frac{3}{2}}-\frac{302946223}{47900160} f_{n+2}+\frac{5348}{14175} f_{n+\frac{5}{2}} \\
-\frac{631808}{467775} f_{n+3}+\frac{167936}{467775} f_{n+\frac{7}{2}}-\frac{1184}{31185} f_{n+4}
\end{array}\right)
\end{aligned}
$$

substituting the equation (47) - (54) into (20) - (27) gives

$$
\begin{aligned}
& y_{n+\frac{1}{2}}^{\prime}=-y_{n}^{\prime}+\frac{1}{2} h y_{n}^{\prime \prime}+\frac{1}{8} h^{2} y_{n}{ }_{n}+h^{3}\left(\begin{array}{l}
\frac{517129}{45619200} f_{n}+\frac{1129981}{53222400} f_{n+\frac{1}{2}}-\frac{1871827}{63866880} f_{n+1} \\
+\frac{5887073}{159667200} f_{n+\frac{3}{2}}-\frac{716363}{21288960} f_{n+2}+\frac{3385541}{159667200} f_{n+\frac{5}{2}} \\
-\frac{2792861}{319334400} f_{n+3}+\frac{22637}{10644480} f_{n+\frac{7}{2}}-\frac{36943}{159667200} f_{n+4}
\end{array}\right) \\
& y_{n+1}^{\prime}=-y_{n}^{\prime}+h y_{n}^{\prime \prime}+\frac{1}{2} h^{2} y_{n}^{\prime \prime \prime}+h^{3}\left(\begin{array}{l}
\frac{286967}{4989600} f_{n}+\frac{32543}{178200} f_{n+\frac{1}{2}}-\frac{22063}{118800} f_{n+1} \\
+\frac{58657}{249480} f_{n+\frac{3}{2}}-\frac{21359}{99792} f_{n+2}+\frac{55969}{415800} f_{n+\frac{5}{2}} \\
-\frac{138317}{2494800} f_{n+3}+\frac{16799}{1247400} f_{n+\frac{7}{2}}-\frac{487}{332640} f_{n+4}
\end{array}\right) \\
& y_{n+\frac{3}{2}}^{\prime}=-y_{n}^{\prime}+\frac{3}{2} h y_{n}^{\prime \prime}+\frac{9}{8} h^{2} y_{n}{ }_{n}+h^{3}\left(\begin{array}{l}
\frac{68769}{492800} f_{n}+\frac{1067877}{1971200} f_{n+\frac{1}{2}}-\frac{1563651}{3942400} f_{n+1} \\
+\frac{32841}{56320} f_{n+\frac{3}{2}}-\frac{419931}{788480} f_{n+2}+\frac{661959}{1971200} f_{n+\frac{5}{2}} \\
-\frac{546129}{3942400} f_{n+3}+\frac{66393}{1971200} f_{n+\frac{7}{2}}-\frac{2889}{788480} f_{n+4}
\end{array}\right) \\
& y_{n+2}^{\prime}=-y_{n}^{\prime}+2 h_{n}{ }_{n}+2 h^{2} y_{n}^{\prime \prime \prime}+h^{3}\left(\begin{array}{l}
\frac{80293}{311850} f_{n}+\frac{57136}{51975} f_{n+\frac{1}{2}}-\frac{18812}{31185} f_{n+1} \\
+\frac{179408}{155925} f_{n+\frac{3}{2}}-\frac{296}{297} f_{n+2}+\frac{98096}{155925} f_{n+\frac{5}{2}} \\
-\frac{40468}{155925} f_{n+3}+\frac{656}{10395} f_{n+\frac{7}{2}}-\frac{2141}{311850} f_{n+4}
\end{array}\right)
\end{aligned}
$$




$$
\begin{aligned}
& y_{n+\frac{5}{2}}^{\prime}=-y_{n}^{\prime}+\frac{5}{2} h y_{n}{ }_{n}+\frac{25}{8} h^{2} y_{n}^{\prime \prime \prime}+h^{3}\left(\begin{array}{l}
\frac{5253125}{12773367} f_{n}+\frac{11851375}{6386688} f_{n+\frac{1}{2}}-\frac{3432125}{4257792} f_{n+1} \\
+\frac{12768625}{6386688} f_{n+\frac{3}{2}}-\frac{19680625}{12773376} f_{n+2}+\frac{307625}{304128} f_{n+\frac{5}{2}} \\
-\frac{5327125}{12773376} f_{n+3}+\frac{647875}{6386688} f_{n+\frac{7}{2}}-\frac{5875}{532224} f_{n+4}
\end{array}\right) \\
& y_{n+3}^{\prime}=-y_{n}^{\prime}+3 h y_{n}^{\prime \prime}+\frac{9}{2} h^{2} y_{n}^{\prime \prime \prime}+h^{3}\left(\begin{array}{l}
\frac{37017}{61600} f_{n}+\frac{6183}{2200} f_{n+\frac{1}{2}}-\frac{6183}{6160} f_{n+1} \\
+\frac{48141}{15400} f_{n+\frac{3}{2}}-\frac{12933}{6160} f_{n+2}+\frac{23787}{15400} f_{n+\frac{5}{2}} \\
-\frac{42691}{4400} f_{n+3}+\frac{459}{3080} f_{n+\frac{7}{2}}-\frac{999}{61600} f_{n+4}
\end{array}\right) \\
& y_{n+\frac{7}{2}}^{\prime}=-y_{n}^{\prime}+\frac{7}{2} h y_{n}{ }_{n}+\frac{49}{8} h^{2} y_{n}^{\prime \prime \prime}+h^{3}\left(\begin{array}{l}
\frac{18850937}{22809600} f_{n}+\frac{30139753}{7603200} f_{n+\frac{1}{2}}-\frac{54639557}{45619200} f_{n+1} \\
+\frac{20689417}{4561920} f_{n+\frac{3}{2}}-\frac{1630279}{608256} f_{n+2}+\frac{52421033}{22809600} f_{n+\frac{5}{2}} \\
-\frac{35782103}{45619200} f_{n+3}+\frac{1570597}{7603200} f_{n+\frac{7}{2}}-\frac{40817}{1824768} f_{n+4}
\end{array}\right) \\
& y_{n+4}^{\prime}=-y_{n}^{\prime}+4 h y_{n}^{\prime \prime}+8 h^{2} y_{n}^{\prime \prime \prime}+h^{3}\left(\begin{array}{l}
\frac{24232}{22275} f_{n}+\frac{828928}{155925} f_{n+\frac{1}{2}}-\frac{72064}{51975} f_{n+1} \\
+\frac{194048}{31185} f_{n+\frac{3}{2}}-\frac{102112}{31185} f_{n+2}+\frac{169472}{51975} f_{n+\frac{5}{2}} \\
-\frac{134528}{155925} f_{n+3}+\frac{51712}{155925} f_{n+\frac{7}{2}}-\frac{296}{10395} f_{n+4}
\end{array}\right)
\end{aligned}
$$

Substituting equation (47) - (54) into (29) - (36) gives

$$
\begin{gathered}
y_{n+\frac{1}{2}}{ }^{\prime} y_{n}{ }_{n}+\frac{1}{2} h y^{\prime \prime \prime}{ }_{n}+h^{2}\left(\begin{array}{l}
\frac{324901}{5806080} f_{n}+\frac{8183}{57600} f_{n+\frac{1}{2}}-\frac{653203}{3628800} f_{n+1}+ \\
\frac{50689}{226800} f_{n+\frac{3}{2}}-\frac{196277}{967680} f_{n+2}+\frac{92473}{725760} f_{n+\frac{5}{2}} \\
-\frac{95167}{1814400} f_{n+3}+\frac{7703}{604800} f_{n+\frac{7}{2}}-\frac{5741}{4147200} f_{n+4}
\end{array}\right) \\
y_{n+1}=y_{n}{ }_{n}+h y_{n}{ }_{n}+h^{2}\left(\begin{array}{l}
\frac{58193}{453600} f_{n}+\frac{7346}{14175} f_{n+\frac{1}{2}}-\frac{81}{200} f_{n+1} \\
+\frac{7729}{14175} f_{n+\frac{3}{2}}-\frac{22703}{45360} f_{n+2}+\frac{1492}{4725} f_{n+\frac{5}{2}} \\
-\frac{14773}{113400} f_{n+3}+\frac{449}{14175} f_{n+\frac{7}{2}}-\frac{521}{151200} f_{n+4}
\end{array}\right)
\end{gathered}
$$




$$
\begin{aligned}
& y_{n+\frac{3}{2}}^{\prime \prime}=y_{n}{ }_{n}+\frac{3}{2} h y_{n}^{\prime \prime \prime}+h^{2}\left(\begin{array}{l}
\frac{71661}{358400} f_{n}+\frac{1467}{1600} f_{n+\frac{1}{2}}-\frac{4707}{11200} f_{n+1}+ \\
\frac{225}{256} f_{n+\frac{3}{2}}-\frac{28143}{35840} f_{n+2}+\frac{11079}{22400} f_{n+\frac{5}{2}} \\
-\frac{9141}{44800} f_{n+3}+\frac{2223}{44800} f_{n+\frac{7}{2}}-\frac{387}{71680} f_{n+4}
\end{array}\right) \\
& y_{n+2}{ }_{n+2} y_{n}+2 h y_{n}+h^{2}\left(\begin{array}{l}
\frac{7703}{28350} f_{n}+\frac{6208}{4725} f_{n+\frac{1}{2}}-\frac{232}{567} f_{n+1} \\
+\frac{20032}{14175} f_{n+\frac{3}{2}}-\frac{47}{45} f_{n+2}+\frac{9536}{14175} f_{n+\frac{5}{2}} \\
-\frac{3944}{14175} f_{n+3}+\frac{64}{28350} f_{n+\frac{7}{2}}-\frac{209}{28350} f_{n+4}
\end{array}\right) \\
& y_{n+\frac{5}{2}}^{\prime \prime}=y_{n}{ }_{n}+\frac{5}{2} h y_{n}{ }^{\prime \prime}+h^{2}\left(\begin{array}{l}
\frac{56975}{165888} f_{n}+\frac{248375}{145152} f_{n+\frac{1}{2}}-\frac{19375}{48384} f_{n+1}+ \\
\frac{5143375}{72576} f_{n+\frac{3}{2}}-\frac{641875}{580608} f_{n+2}+\frac{225}{256} f_{n+\frac{5}{2}} \\
-\frac{12875}{36288} f_{n+3}+\frac{3125}{36288} f_{n+\frac{7}{2}}-\frac{3625}{387072} f_{n+4}
\end{array}\right) \\
& y_{n+3}=y_{n}{ }_{n}+3 h y_{n}{ }_{n}+h^{2}\left(\begin{array}{l}
\frac{93}{224} f_{n}+\frac{369}{175} f_{n+\frac{1}{2}}-\frac{549}{1400} f_{n+1}+\frac{444}{175} f_{n+\frac{3}{2}}- \\
\frac{639}{560} f_{n+2}+\frac{9}{7} f_{n+\frac{5}{2}}-\frac{81}{200} f_{n+3}+\frac{18}{175} f_{n+\frac{7}{2}}-\frac{9}{800} f_{n+4}
\end{array}\right) \\
& y_{n+\frac{7}{2}}^{\prime \prime}=y_{n}+\frac{7}{2} h y_{n}{ }_{n}+h^{2}\left(\begin{array}{l}
\frac{2019731}{4147200} f_{n}+\frac{216433}{86400} f_{n+\frac{1}{2}}-\frac{98441}{259200} f_{n+1}+ \\
\frac{1601467}{518400} f_{n+\frac{3}{2}}-\frac{160867}{138240} f_{n+2}+\frac{55223}{32400} f_{n+\frac{5}{2}} \\
-\frac{127253}{518400} f_{n+3}+\frac{8183}{57600} f_{n+\frac{7}{2}}-\frac{57281}{4147200} f_{n+4}
\end{array}\right) \\
& y_{n+4}=y_{n}{ }_{n}+4 h y{ }_{n}+h^{2}\left(\begin{array}{l}
\frac{7912}{14175} f_{n}+\frac{5888}{2025} f_{n+\frac{1}{2}}-\frac{1856}{4725} f_{n+1}+\frac{10496}{2835} f_{n+\frac{3}{2}}- \\
\frac{3632}{2835} f_{n+2}+\frac{10496}{4725} f_{n+\frac{5}{2}}-\frac{1856}{14175} f_{n+3}+\frac{5888}{14175} f_{n+\frac{7}{2}}
\end{array}\right)
\end{aligned}
$$

Substituting equation (47) - (54) into (38) - (45) gives

$$
y_{n+\frac{1}{2}}^{\prime \prime \prime}=y_{n}^{\prime \prime \prime}+h\left(\begin{array}{l}
\frac{1070017}{7257600} f_{n}+\frac{2233547}{3628800} f_{n+\frac{1}{2}}-\frac{2302297}{3628800} f_{n+1}+\frac{2797679}{3628800} f_{n+\frac{3}{2}}-\frac{31457}{45360} f_{n+2}+ \\
\frac{1573169}{3628800} f_{n+\frac{5}{2}}-\frac{645607}{3628800} f_{n+3}+\frac{156437}{3628800} f_{n+\frac{7}{2}}-\frac{33953}{7257600} f_{n+4}
\end{array}\right)
$$




$$
\begin{aligned}
& y_{n+1}^{\prime \prime \prime}=y_{n}{ }_{n}+h\left(\begin{array}{l}
\frac{32377}{226800} f_{n}+\frac{22823}{28350} f_{n+\frac{1}{2}}-\frac{21247}{113400} f_{n+1}+\frac{15011}{28350} f_{n+\frac{3}{2}}-\frac{2903}{5670} f_{n+2}+ \\
\frac{9341}{28350} f_{n+\frac{5}{2}}-\frac{15577}{113400} f_{n+3}+\frac{953}{28350} f_{n+\frac{7}{2}}-\frac{119}{32400} f_{n+4}
\end{array}\right) \\
& y_{n+\frac{3}{2}}^{\prime \prime \prime}=y_{n}^{\prime \prime \prime}+h\left(\begin{array}{l}
\frac{12881}{89600} f_{n}+\frac{35451}{44800} f_{n+\frac{1}{2}}+\frac{719}{44800} f_{n+1}+\frac{39967}{44800} f_{n+\frac{3}{2}}-\frac{351}{560} f_{n+2}+ \\
\frac{17217}{44800} f_{n+\frac{5}{2}}-\frac{7031}{44800} f_{n+3}+\frac{243}{6400} f_{n+\frac{7}{2}}-\frac{369}{89600} f_{n+4}
\end{array}\right) \\
& y_{n+2}^{\prime \prime \prime}=y_{n}^{\prime \prime \prime}+h\left(\begin{array}{l}
\frac{4063}{28350} f_{n}+\frac{11288}{14175} f_{n+\frac{1}{2}}+\frac{122}{14175} f_{n+1}+\frac{16376}{14175} f_{n+\frac{3}{2}}-\frac{908}{2835} f_{n+2}+ \\
\frac{4616}{14175} f_{n+\frac{5}{2}}-\frac{1978}{14175} f_{n+3}+\frac{488}{14175} f_{n+\frac{7}{2}}-\frac{107}{28350} f_{n+4}
\end{array}\right) \\
& { }^{\prime \prime \prime}{ }_{n+\frac{5}{2}}=y_{n}^{\prime \prime \prime}+h\left(\begin{array}{l}
\frac{41705}{290304} f_{n}+\frac{115075}{145152} f_{n+\frac{1}{2}}+\frac{3775}{145152} f_{n+1}+\frac{159175}{145152} f_{n+\frac{3}{2}}-\frac{125}{9072} f_{n+2}+ \\
\frac{85465}{145152} f_{n+\frac{5}{2}}-\frac{24575}{145152} f_{n+3}+\frac{5725}{145152} f_{n+\frac{7}{2}}-\frac{175}{41472} f_{n+4}
\end{array}\right) \\
& y_{n+3}^{\prime \prime \prime}=y_{n}^{\prime \prime \prime}+h\left(\begin{array}{l}
\frac{401}{2800} f_{n}+\frac{279}{350} f_{n+\frac{1}{2}}+\frac{9}{1400} f_{n+1}+\frac{403}{350} f_{n+\frac{3}{2}}-\frac{9}{70} f_{n+2}+ \\
\frac{333}{350} f_{n+\frac{5}{2}}+\frac{79}{1400} f_{n+3}+\frac{9}{350} f_{n+\frac{7}{2}}-\frac{9}{2800} f_{n+4}
\end{array}\right) \\
& y_{n+\frac{7}{2}}^{\prime \prime \prime}=y_{n}^{\prime \prime}+h\left(\begin{array}{l}
\frac{149527}{1036800} f_{n}+\frac{408317}{518400} f_{n+\frac{1}{2}}+\frac{24353}{518400} f_{n+1}+\frac{542969}{518400} f_{n+\frac{3}{2}}+\frac{343}{6480} f_{n+2}+ \\
\frac{368039}{518400} f_{n+\frac{5}{2}}+\frac{261023}{518400} f_{n+3}+\frac{111587}{518400} f_{n+\frac{7}{2}}-\frac{8183}{1036800} f_{n+4}
\end{array}\right) \\
& y_{n+4}^{\prime \prime \prime}=y_{n}^{\prime \prime \prime}+h\left(\begin{array}{l}
\frac{1978}{14175} f_{n}+\frac{11776}{14175} f_{n+\frac{1}{2}}-\frac{1856}{14175} f_{n+1}+\frac{20992}{14175} f_{n+\frac{3}{2}}-\frac{21816}{2835} f_{n+2}+ \\
\frac{20992}{14175} f_{n+\frac{5}{2}}-\frac{1856}{14175} f_{n+3}+\frac{11776}{14175} f_{n+\frac{7}{2}}+\frac{1978}{14175} f_{n+4}
\end{array}\right)
\end{aligned}
$$

\section{Basic Properties of the Methods}

Here, we present the basic properties of the new numerical method which include order and error constant, consistency, convergence and zero stability.

\subsection{Consistency of the Numerical Method}

A numerical method is said to be consistence if the following are satisfies.

i. The order of the scheme must be greater than or equal to 1 i.e. $\mathrm{p} \geq 1$. 

ii. $\sum_{j=0}^{k} \alpha_{j}=0, \alpha_{j}$ 's are the coefficients of the first characteristic polynomial
iii. $\rho(r)=\rho^{\prime}(r)=0$ where $r=1$
iv. $\rho^{\text {iv }}(r)=4 ! \sigma(r)$ for $r=1, \sigma(\mathrm{r})$ is the second characteristic polynomial.

According to [7] the first condition is a sufficient condition for the associated block method to be consistent. To obtain the consistency, we used the above conditions;

\subsection{Consistency of the Proposed Method}

Consider the new developed method (11)

$$
y_{n+4}+140 y_{n+1}-120 y_{n+\frac{1}{2}}-56 y_{n+\frac{3}{2}}+35 y_{n}=\frac{h^{4}}{829440}\left[\begin{array}{c}
-565 f_{n}+298040 f_{n+\frac{1}{2}}+1409780 f_{n+1}+ \\
1022792 f_{n+\frac{3}{2}}+615410 f_{n+2}+192200 f_{n+\frac{5}{2}} \\
+86420 f_{n+3}+4280 f_{n+\frac{7}{2}}+443 f_{n+4}
\end{array}\right]
$$

i. The order, $\mathrm{p}=10$ hence it satisfies $\mathrm{p} \geqslant 1$

ii. $\sum_{j=0}^{4} \alpha_{j}=\alpha_{0}+\alpha_{\frac{1}{2}}+\alpha_{1}+\alpha_{\frac{3}{2}}+\alpha_{4}=35-120+140-56+1=0$

iii. $\rho(r)=r^{4}+140 r^{1}-120 r^{\frac{1}{2}}-56 r^{\frac{3}{2}}+35 r^{0}$.

$$
\begin{gathered}
\rho(r)=r^{4}+140 r^{1}-120 r^{\frac{1}{2}}-56 r^{\frac{3}{2}}+35 \\
\rho(1)=(1)^{4}+140(1)^{1}-120(1)^{\frac{1}{2}}-56(1)^{\frac{3}{2}}+35 \nsucceq 0 \\
\rho^{\prime}(r)=4 r^{3}+140-60 r^{\frac{-1}{2}}-84 r^{-\frac{1}{2}} \\
\rho^{\prime}(1)=(1)^{3}+140-60(1)^{\frac{-1}{2}}-84(1)^{-\frac{1}{2}} \nsucceq 0
\end{gathered}
$$

Since $\mathrm{p}(1)=\rho^{\prime}(r)=0$, this shows that condition is satisfied

iv. By the main method, the first and second characteristics polynomial of the method are

$$
\begin{gathered}
p(r)=r^{4}+140 r^{1}-120 r^{\frac{1}{2}}-56 r^{\frac{3}{2}}+35 r^{0}=r^{4}+140 r^{1}-120 r^{\frac{1}{2}}-56 r^{\frac{3}{2}}+35 \\
\sigma(r)=\frac{-565 r^{0}}{829440}+\frac{298040 r^{\frac{1}{2}}}{829440}+\frac{1409780 r^{1}}{829440}+\frac{1022792 r^{\frac{3}{2}}}{829440}+\frac{615410 r^{2}}{829440}+\frac{192200 r^{\frac{5}{2}}}{829440}+\frac{86420 r^{3}}{829440}+\frac{4280 r^{\frac{7}{2}}}{829440}+\frac{443 r^{4}}{829440} \\
=\frac{-565}{829440}+\frac{298040 r^{\frac{1}{2}}}{829440}+\frac{1409780 r^{1}}{829440}+\frac{1022792 r^{\frac{3}{2}}}{829440}+\frac{615410 r^{2}}{829440}+\frac{192200 r^{\frac{5}{2}}}{829440}+\frac{86420 r^{3}}{829440}+\frac{4280 r^{\frac{7}{2}}}{829440}+\frac{443 r^{4}}{829440} \\
\rho^{\prime}(r)=4 r^{3}+140-60 r^{\frac{-1}{2}}-84 r^{-\frac{1}{2}} \\
\rho^{\prime \prime}(r)=12 r^{2}+30 r^{\frac{-3}{2}}-42 r^{\frac{-1}{2}} \\
p(r)^{\prime \prime \prime}=24 r-45 r^{\frac{-5}{2}}+21 r^{\frac{-3}{2}} \\
p(r)^{\text {iv }}=24+\frac{225}{2}(r)^{-\frac{7}{2}}-\frac{63}{2}(r)^{\frac{-5}{2}} \\
\text { Putting r }=1
\end{gathered}
$$




$$
\begin{gathered}
p(1)^{\mathbf{i v}}=24+\frac{225}{2}(1)^{-\frac{7}{2}}-\frac{63}{2}(1)^{\frac{-5}{2}}=105 \\
\sigma(r)=\frac{-565}{829440}+\frac{298040 r^{\frac{1}{2}}}{829440}+\frac{1409780 r^{1}}{829440}+\frac{1022792 r^{\frac{3}{2}}}{829440}+\frac{615410 r^{2}}{829440}+\frac{192200 r^{\frac{5}{2}}}{829440}+\frac{86420 r^{3}}{829440}+\frac{4280 r^{\frac{7}{2}}}{829440}+\frac{443 r^{4}}{829440} \\
\sigma(1)=\frac{1}{829440}[-565+298040+1409780+1022792+615410+192200+86420+4280+443]=\frac{3628800}{8299440} \\
4 ! \sigma(1)=4 ! * \frac{4375}{1000}=105 \\
p(1)^{\mathbf{i v}}=4 ! \sigma(1)=105
\end{gathered}
$$

For principal root $\mathrm{r}=1$, the conditions (1)-(iv) above are satisfied. Hence the Method is Consistence.

\subsection{Zero Stability of Block Method}

Given the general form of block method as

$$
A^{(0)} Y_{m}=A^{(i)} Y_{m-1}+h^{\mu}\left[B^{(i)} F_{m}+B^{(0)} F_{m-1}\right]
$$

A block method is said to be zero stable, if the roots

$$
\operatorname{det}\left[\lambda A^{(0)}-A^{(i)}\right]=0
$$

of the first characteristic polynomial satisfy $|\lambda| \leq 1$ and for the roots with $|\lambda| \leq 1$, the multiplicity must not exceed the order of the differential equation.

\subsection{Zero Stability of the Proposed Block Method}

Given the general form of block method as

$$
\begin{gathered}
\left.A=\left[\begin{array}{llllllll}
1 & 0 & 0 & 0 & 0 & 0 & 0 & 0 \\
0 & 1 & 0 & 0 & 0 & 0 & 0 & 0 \\
1 & 0 & 1 & 0 & 0 & 0 & 0 & 0 \\
0 & 0 & 0 & 1 & 0 & 0 & 0 & 0 \\
0 & 0 & 0 & 0 & 1 & 0 & 0 & 0 \\
0 & 0 & 0 & 0 & 0 & 1 & 0 & 0 \\
0 & 0 & 0 & 0 & 0 & 0 & 1 & 0 \\
0 & 0 & 0 & 0 & 0 & 0 & 0 & 1
\end{array}\right]-\left[\begin{array}{llllllll}
0 & 0 & 0 & 0 & 0 & 0 & 0 & 1 \\
0 & 0 & 0 & 0 & 0 & 0 & 0 & 1 \\
0 & 0 & 0 & 0 & 0 & 0 & 0 & 1 \\
0 & 0 & 0 & 0 & 0 & 0 & 0 & 1 \\
0 & 0 & 0 & 0 & 0 & 0 & 0 & 1 \\
0 & 0 & 0 & 0 & 0 & 0 & 0 & 1 \\
0 & 0 & 0 & 0 & 0 & 0 & 0 & 1 \\
0 & 0 & 0 & 0 & 0 & 0 & 0 & 1
\end{array}\right]\right]=0 \\
z
\end{gathered}
$$

Hence the block is zero stable.

\subsection{Convergence}

Theorem 1: Convergence [10-11]

The necessary and sufficient condition for a linear multistep method to be convergent is for it to be consistent and zero stable. From the theorem above, the blocks are convergent.

\section{Numerical Experiment}

The mode of implementation of our method is by combining the block integrators (47 - 78) to simultaneously solve the system of equations of initial value problems of fourth order Ordinary differential equations without requiring starting values and predictors.

\subsection{Numerical Example}

Consider the linear system below, solved by erudite scholar

\begin{tabular}{|c|c|c|c|c|}
\hline$x$ & y-Exact Result & y-Computed Result & y-Error & y-Time \\
\hline 0.1 & 0.9048374180359595 & 0.904837418035959520 & $0.000000 \mathrm{e}+00$ & 0.0014 \\
\hline 0.2 & 0.8187307530779818 & 0.818730753077981930 & $1.110223 \mathrm{e}-16$ & 0.0020 \\
\hline 0.3 & 0.7408182206817178 & 0.740818220681717880 & $1.110223 \mathrm{e}-16$ & 0.0022 \\
\hline 0.4 & 0.6703200460356393 & 0.670320046035639330 & $0.000000 \mathrm{e}+00$ & 0.0023 \\
\hline 0.5 & 0.6065306597126334 & 0.606530659712633540 & $1.110223 \mathrm{e}-16$ & 0.0024 \\
\hline 0.6 & 0.5488116360940264 & 0.548811636094026390 & $0.000000 \mathrm{e}+00$ & 0.0026 \\
\hline
\end{tabular}
Kasimet al (2015)

$$
\begin{array}{ll}
y^{i v}=e^{3 x} u, & y(0)=1, y^{\prime}(0)=-1, y^{\prime \prime}(0)=1, y^{\prime \prime}(0)=-1 \\
z^{i v}=16 e^{-x} y, & z(0)=1, z^{\prime}(0)=-2, z^{\prime \prime}(0)=4, z^{\prime \prime}(0)=-8 \\
w^{i v}=81 e^{-x} z, & w(0)=1, w^{\prime}(0)=-3, w^{\prime \prime}(0)=9, w^{\prime \prime \prime}(0)=-27 \\
u^{i v}=256 e^{-x} w, & u(0)=1, u^{\prime}(0)=-4, u^{\prime \prime}(0)=16, u^{\prime \prime \prime}(0)=-64
\end{array}
$$

The exact solution is given by

$$
\begin{array}{ll}
y=e^{-x} & z=e^{-2 x} \\
w=e^{-3 x} & u=e^{-4 x}
\end{array}
$$

The problem is integrated in the interval $[0,3]$

\subsection{Numerical Results}

The numerical results of the proposed method are presented in Table 1 - Table 4 below.

Table 1. The y-exact Result, y-computed results and absolute error in the new method. 


\begin{tabular}{cllll}
\hline$x$ & y-Exact Result & y-Computed Result & y-Error & y-Time \\
\hline 0.7 & 0.4965853037914095 & 0.496585303791409580 & $1.110223 \mathrm{e}-16$ & 0.0027 \\
0.8 & 0.4493289641172216 & 0.449328964117221900 & $3.330669 \mathrm{e}-16$ & 0.0028 \\
0.9 & 0.4065696597405991 & 0.406569659740599500 & $3.885781 \mathrm{e}-16$ & 0.0029 \\
1.0 & 0.3678794411714423 & 0.367879441171442890 & $5.551115 \mathrm{e}-16$ & 0.0031 \\
\hline
\end{tabular}

Table 2. The z-exact solutions, z-computed results and absolute error in the developed method.

\begin{tabular}{cllll}
\hline$x$ & z-Exact Result & z-Computed Result & z-Error & z-Time \\
\hline 0.1 & 0.8187307530779818 & 0.818730753077985710 & $3.885781 \mathrm{e}-15$ & 0.0409 \\
0.2 & 0.6703200460356393 & 0.670320046035695950 & $5.662137 \mathrm{e}-14$ & 0.0445 \\
0.3 & 0.5488116360940264 & 0.548811636094255100 & $2.287059 \mathrm{e}-13$ & 0.0471 \\
0.4 & 0.4493289641172216 & 0.449328964117811200 & $5.896394 \mathrm{e}-13$ & 0.0498 \\
0.5 & 0.3678794411714423 & 0.367879441172651030 & $1.208700 \mathrm{e}-12$ & 0.0525 \\
0.6 & 0.3011942119122020 & 0.301194211914357360 & $2.155331 \mathrm{e}-12$ & 0.0556 \\
0.7 & 0.2465969639416064 & 0.246596963945105550 & $3.499118 \mathrm{e}-12$ & 0.0579 \\
0.8 & 0.2018965179946554 & 0.201896517999964550 & $5.309170 \mathrm{e}-12$ & 0.0613 \\
0.9 & 0.1652988882215865 & 0.165298888229241880 & $7.655349 \mathrm{e}-12$ & 0.0637 \\
1.0 & 0.1353352832366127 & 0.135335283247220110 & $1.060740 \mathrm{e}-11$ & 0.0660 \\
\hline
\end{tabular}

Table 3. The w-exact solutions, w-computed results and absolute error in the developed method.

\begin{tabular}{cllll}
\hline$x$ & w-Exact Result & w-Computed Result & w-Error & w-Time \\
\hline 0.1 & 0.7408182206817178 & 0.740818220682830760 & $1.112999 \mathrm{e}-12$ & 0.0407 \\
0.2 & 0.5488116360940264 & 0.548811636109987180 & $1.596079 \mathrm{e}-11$ & 0.0444 \\
0.3 & 0.4065696597405991 & 0.406569659805117440 & $6.451839 \mathrm{e}-11$ & 0.0471 \\
0.4 & 0.3011942119122020 & 0.301194212078471410 & $1.662694 \mathrm{e}-10$ & 0.0493 \\
0.5 & 0.2231301601484298 & 0.223130160489256490 & $3.408267 \mathrm{e}-10$ & 0.0529 \\
0.6 & 0.1652988882215865 & 0.165298888829352730 & $6.077662 \mathrm{e}-10$ & 0.0556 \\
0.7 & 0.1224564282529819 & 0.122456429239647950 & $9.866660 \mathrm{e}-10$ & 0.0579 \\
0.8 & 0.0907179532894125 & 0.090717954786547317 & $1.497135 \mathrm{e}-09$ & 0.0606 \\
0.9 & 0.0672055127397498 & 0.067205514898420304 & $2.158671 \mathrm{e}-09$ & 0.0630 \\
1.0 & 0.0497870683678639 & 0.049787071359048696 & $2.991185 \mathrm{e}-09$ & 0.0661 \\
\hline
\end{tabular}

Table 4. The u-exact solutions, $u$-computed results and absolute error in the developed method.

\begin{tabular}{cllll}
\hline$x$ & u-Exact Result & u-Computed Result & u-Error & u-Time \\
\hline 0.1 & 0.6703200460356393 & 0.670320046089736500 & $5.409717 \mathrm{e}-11$ & 0.0423 \\
0.2 & 0.4493289641172216 & 0.449328964892583670 & $7.753621 \mathrm{e}-10$ & 0.0459 \\
0.3 & 0.3011942119122020 & 0.301194215046340250 & $3.134138 \mathrm{e}-09$ & 0.0494 \\
0.4 & 0.2018965179946554 & 0.201896526071894570 & $8.077239 \mathrm{e}-09$ & 0.0518 \\
0.5 & 0.1353352832366127 & 0.135335299794267390 & $1.655765 \mathrm{e}-08$ & 0.0547 \\
0.6 & 0.0907179532894125 & 0.090717982816007847 & $2.952660 \mathrm{e}-08$ & 0.0575 \\
0.7 & 0.0608100626252180 & 0.060810110560611363 & $4.793539 \mathrm{e}-08$ & 0.0597 \\
0.8 & 0.0407622039783662 & 0.040762276715137880 & $7.273677 \mathrm{e}-08$ & 0.0646 \\
0.9 & 0.0273237224472926 & 0.027323827325754557 & $1.048785 \mathrm{e}-07$ & 0.0670 \\
1.0 & 0.0183156388887342 & 0.018315784215550579 & $1.453268 \mathrm{e}-07$ & 0.0697 \\
\hline
\end{tabular}

\section{Discussion of the New Method and Results}

This work presents the derivation of a new numerical method for solving fourth order ordinary differential equation. The method makes use of power series as the approximate solution to (1). The method is fully implicit in nature with four-hybrid points which were carefully selected with equal interval within four-step. The method has order of accuracy $\mathrm{P}=10$, using Taylor series as established in [7, 13]. The method is consistence, convergent and zero stable. The method was applied to solve linear system of equations. The Results were displayed in Table 1 - Table 4.

\section{Conclusion and Recommendation of the New Method}

In this study, we discussed in details, the derivation, and analysis of a new numerical method for solving fourth order ordinary differential equations by hybrid block techniques. Power series was used as basic function. The method has four grid points and 4-off grid points as it has been chosen carefully within the interval of integration of four-step. The main purpose is to introduce a new numerical method as a recommendation of numerical approximate values which may be agreed or tends to some existing method of solution of various initial value problems of fourth order ordinary differential equations. We have been able to establish the new proposed method by the Results in Table $1-$ Table 4 . All the 
mathematical formulations were carried out by using Maple Mathematical Software. In future, we will attempt to check the authenticity of this method on boundary value problem with numerous complex examples.

\section{References}

[1] Familua, A. B. and Omole, E. O. (2017) Five points Mono Hybrid point Linear Multistep Method for solving nth Order Ordinary Differential Equations Using Power Series function. Asian Journal of Research and Mathematics, 3 (1), 1-17. http://dx.doi.org/10.9734/ARJOM/2017/31190.

[2] Kayode, S. J, Ige, S. O, Obarhua, F. O. and Omole, E. O. (2018) An Order Six Stormer-cowell-type Method for Solving Directly Higher Order Ordinary Differential Equations. Asian Research Journal of Mathematics, 11 (3), 1-12. http://dx.doi.org/10.9734/ARJOM/2018/44676.

[3] Adoghe, L. O, Ogunware, B. G. and Omole, E. O. (2016) A family of symmetric implicit higher order methods for the solution of third order initial value problems in ordinary differential equations. Journal of Theoretical Mathematics \& Application, 6 (3): 67-84.

[4] Areo, E. A. and Omole, E. O. (2015) HALF-Step symmetric continuous hybrid block method for the numerical solutions of fourth order ordinary differential equations, Archives of Applied Science Research, 7 (10): 39-49. www.scholarsresearchlibrary.com.

[5] Sunday, J. Skwame Y. and Huoma I. U. (2015) Implicit One-Step Legendre Polynomial Hybrid Block Method for the Solution of First-Order Stiff Differential Equations. British Journal of Mathematics and Computer Science. 8 (6), 482-491, https://doi.org/10.9734/BJMCS/2015/16252.

[6] Al-Mazmumy, M. Al-Mutairi, A. and Al-Zahrani, K. (2017). An Efficient Decomposition Method for Solving Bratu's Boundary Value Problem. American Journal of
Computational Mathematics, 7, 84-93. https://doi.org/10.4236/ajcm.2017.71007

[7] Atkinson, K. E. (1989) An Introduction to Numerical Analysis," 2nd Edition, John Wiley and Sons, New York, pp- 284.

[8] M. I. Bhatti, and Bracken, P. (2007) Solutions of Differential Equations in a Bernstein Polynomial Basis. Journal of Computational and Applied Mathematics, Vol. 205, No. 1, pp. 272-280. doi: 10.1016/j.cam.2006.05.002

[9] Adeniran, A. O. \& Longe, I. O. (2019) Solving directly second order initial value problems with Lucas polynomial. Journal of Advances in Mathematics and Computer science, 32 (4), 1-7. http://dx.doi.org/10.9734/jamcs/2019/v32i430152.

[10] R. L. Burden, and J. D. Faires, (1992) Numerical Analysis," Brooks/Cole Publishing Co., Pacific Grove.

[11] Fatunla, S. O. (1991) Block Method for Second Order IVPs. International Journal of Computer Mathematics, 41, 55-63, http://dx.doi.org/10.1080/00207169108804026.

[12] Buckmire, R. (2003) Investigations of Nonstandard, Mickens-Type, Finite-Difference Schemes for Singular Boundary Value Problems in Cylindrical or Spherical Coordinate. Numerical Methods for Partial Differential Equations, 19 , 380-398. https://doi.org/10.1002/num.10055.

[13] Lambert, J. D.(1973) Computational Methods in ODEs. John Wiley \& Sons, New York.

[14] Harier, and G. Wanner, (1996) Solving Ordinary Differential Equations II, Stiff and Differential-Algebraic Problems, Springer-Verlag, New York. doi: 10.1007/978-3-642-05221-7.

[15] Kasim H, Fudziah I. and Norazak S. (2015) Two Embedded Pairs of Runge-Kutta Type Methods for Direct Solution of Special Fourth-Order Ordinary Differential Equations, Journal of Mathematical Problems in Engineering, Vol. 15, Article ID 196595, 12 pages. 